\title{
The Verification of TDFAM using Freeway Flow Counts
}

\author{
Yu-Chun Liao, Associate Professor \\ Department of Civil Engineering, Chung Yuan Christian \\ University \\ Taoyuan, Taiwan \\ yuchun@cycu.edu.tw
}

\begin{abstract}
The previous study of the time-dependent modification of Fluid Analogy Method (TDFAM) model was characterized by key parameters and performance variability, and presented as a sound alternative for O-D estimation with the absence of historical matrix. This study verified the model performance with empirical data of flow counts on freeway ramps and the estimation outcomes showed acceptable errors and calculation efficiency before applying calibration procedure
\end{abstract} for error reduction.

\section{Keywords - TDFAM, O-D estimation, freeway ramps, flow counts}

\section{Introduction}

The origin-destination traffic flow information was conventionally used for long-term transportation planning and management, as well as an important basis for policy analysis. Such information was traditionally obtained from survey data or derived from simulation models. However, the O-D information was difficult and costly to collect in general, let along the survey scale presented another serious issue.

In the near past, the process of estimating O-D flows changed gradually from static to dynamic approaches due to the requirement of better foreseeing the main corridor traffic and the short-term management strategies. For example, Willumsen [1] brought up the concept of changeable O-D flows with time-varying modification of the Maximum Entropy Model by dividing the time period into numerous intervals to make estimate conveniently. The static model was

\author{
Chi-Hao Lin, Doctoral Candidate \\ Department of Civil Engineering, Chung Yuan Christian \\ University \\ Taoyuan, Taiwan \\ g9502201@cycu.edu.tw
}

therefore transformed into the quasi-dynamic edition without largely re-formulation. Since then, the later studies frequently followed the concept of using multiple time segments for modification on static models. Among those, several studies [2][3][4][5] of modification models aimed to estimate the ramp out-flows of freeway corridors with varying time spans, and to seek strategies of alleviating congestion due to heavy traffic on ramps.

Acknowledging the survey difficulties and the dynamic requirement as mentioned, the previous research of this study was a series attempts trying to estimate O-D flows with the absence of historical O-D matrix. Models were built based on the concept of Fluid Analogy Method (FAM) originally applying on estimating bus passenger O-D trips [6]. The series started by Kao's attempt [7] of transforming FAM to estimate O-D flows on the access-controlled freeway corridor.

The adaptability problems of the transformation were soon realized that the bus-passenger version was a static model with space-dimension only, and the time-related freeway on the contrary presented the dynamic characteristics posing difficulties for model re-formation. Nonetheless, the previous studies [8] of this series obtained progressive outcomes and experiences of re-formulating FAM with explicit time-dimension parameters, and Kao's FAM model was thereafter re-derived as the time-dependent modification for FAM model (TDFAM) shown as Fig.1 with structure of multi-layer matrices. 


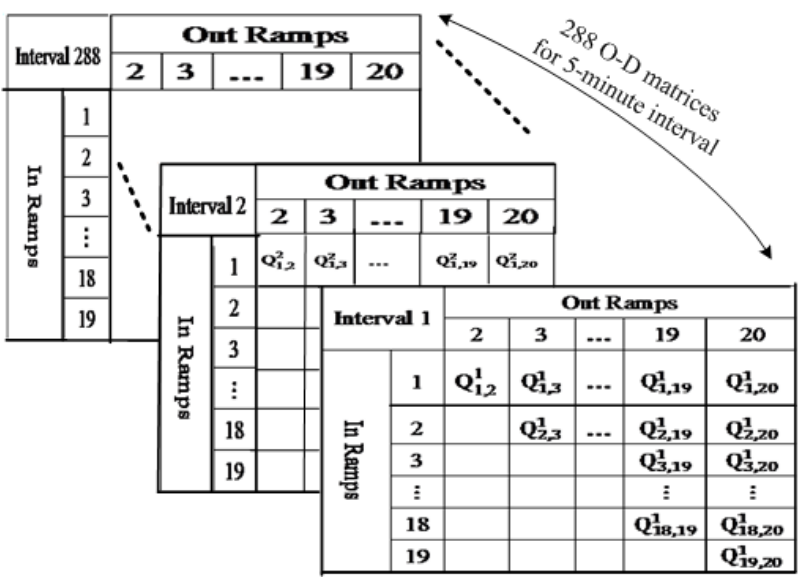

Fig.1 The 288 O-D matrices for 5-minute interval

The merit of the TDFAM used in-flow and out-flow data of freeway ramps to estimate O-D flows without executing traditional full-scale equilibrium assignment. By using simulation data, key parameters and performance variability of TDFAM were explored intensively [8], and the estimation outcomes were satisfying before applying calibration procedure for error reduction. Based on the practice findings, TDFAM presented a sound alternative for estimating O-D flows with the absence of historical matrix. This study is to verify the model performance of TDFAM with empirical data as the prerequisite of further spatial studies and freeway management.

The verification was conducted by using time-dependent (time interval is 5 minutes) in-flow and out-flow data of Sun Yat-Sen Freeway ramps firstly to estimate time-dependent O-D distributions for each interval, followed by re-calculating time-dependent off-ramp flows for error evaluation. The northern part of the freeway covers the service among major urban areas with $40 \%$ of the island population, substantial industrial activities as well as intensive allocation of freeway interchanges. Users of the northern area access the freeway through about 20 ramps which are the target ends for modeling. The real-time flow data are collected every 5-minute interval by detectors. The application of TDFAM model on this freeway section with large size data, multi-layer matrices, acceptable errors and fast calculation shows the practicability of this methodology.

\section{The analysis and results}

The study used Excel-VBA to organize original 5-minute-interval ramp flow counts and to aggregate data into various time intervals, including 30 and 60 minutes (48 and 24 matrices respectively). Total elapse of 48 hours was used for 24-hour analysis span to mitigate spatial-temporal open condition problem of flow convergence. The estimation was processed on the computer equipped with Intel (R) core (TM) i5-2320 CPU, 3.00GMz, 4GB RAM and 500G hard discs.

After time-dependent matrices estimated and ramp flows derived to compare with detector counts, total errors were calculated for both 30-minute and 60-minute intervals. Shown as Fig.2, in general, two types of intervals did not differentiate each other significantly according to error comparison of derived ramp flows.

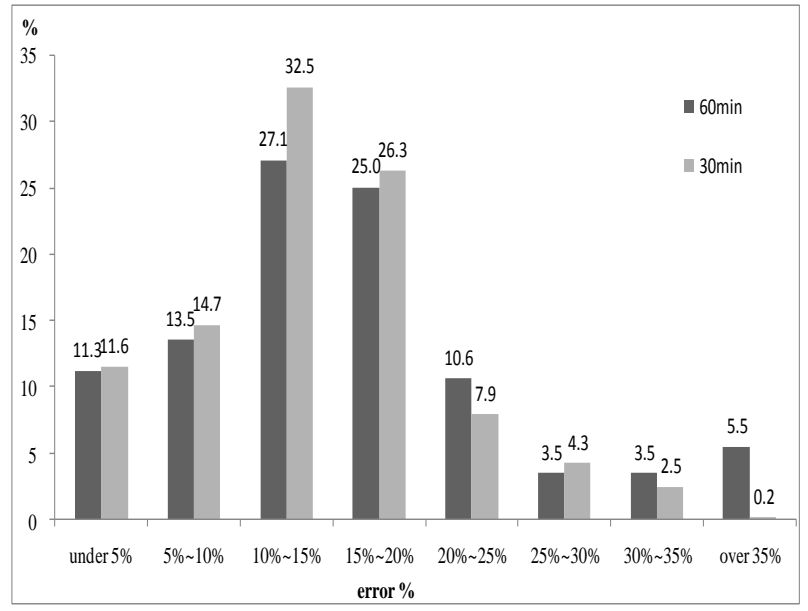

Fig. 2 The error distribution for 30-minute and 60-minute interval

In other words, though the small-interval matrices better estimated time varied O-D information, nevertheless the verification by link flows likely could be done by large time frame. For error distribution, $32.5 \%$ of the errors were between $10 \%$ to $15 \%$, followed by $26.3 \%$ of estimating errors 
between $15 \%$ to $20 \%$. Overall, more than $80 \%$ of errors of ramp flow comparison were less than $20 \%$.

The running time of TDFAM model processed for O-D estimation was less than one minute as Table 1 . In addition, the study also checked the run time for 5-minute interval (288 matrices); it showed less than 10 minutes with intermediate data access during processing and less than 5 minutes without intermediate access. Overall, the calculation efficiency of TDFAM model was substantially acceptable.

Table 1. The comparison of running time

\begin{tabular}{||c|c|c|c|c||}
\hline \hline \multirow{2}{*}{$\begin{array}{c}\text { Size of } \\
\text { interval }\end{array}$} & \multicolumn{3}{|c||}{ Running time } & \multirow{2}{*}{ Reduce } \\
\cline { 2 - 4 } & access & $\begin{array}{c}\text { Second } \\
(\mathrm{s})\end{array}$ & $\begin{array}{c}\text { Minute } \\
(\mathrm{min})\end{array}$ & \\
\hline \multirow{2}{*}{$30 \mathrm{~min}$} & Yes & 40.1 & 0.67 & \multirow{2}{*}{86.78} \\
\cline { 2 - 4 } & No & 5.3 & 0.09 & \\
\hline \multirow{2}{*}{$60 \mathrm{~min}$} & yes & 19.5 & 0.33 & \multirow{2}{*}{80.00} \\
\cline { 2 - 4 } & no & 3.9 & 0.07 & \\
\hline
\end{tabular}

However, error distribution could be mis-pictured due to small ramp flow counts with large error percentage. Fig.4 and Fig.5 showed weekday/weekend error distribution coupled by ramp flows for closer examination. For ramps representing heavy, median and low volume of flows, heavy-volume ramp revealed reasonable estimation errors compared to others; error distributions vary moderately compared to volume peaking vibration. The errors were expected to be reduced by re-distribution process; however, the calibration methodology has not been constructed due to the complex structure of simultaneous matrices calculation.

Other studies for O-D estimation with simulation data reported similar error ranges between 5\% 20\% [3][5] mostly with error calibration.

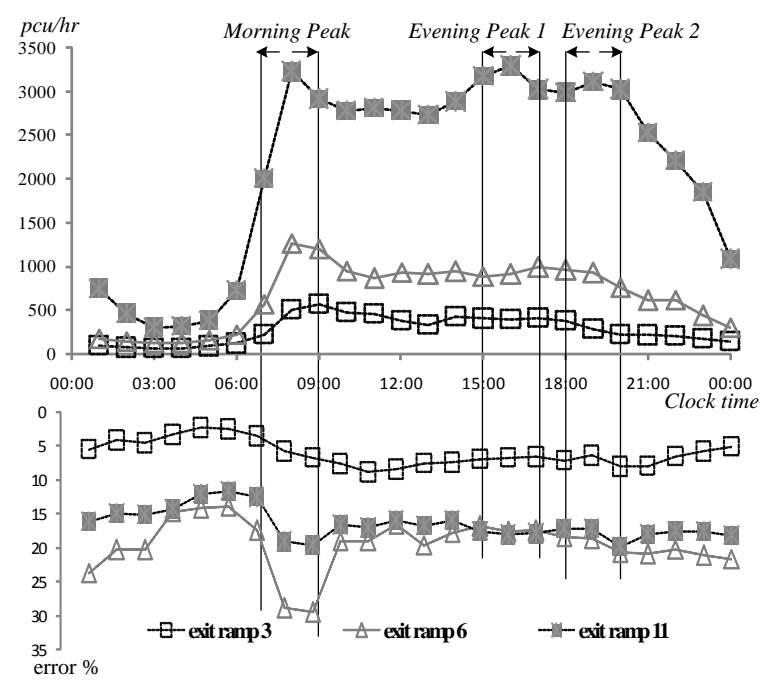

Fig.4 The error distribution and off-ramp flow counts for weekday

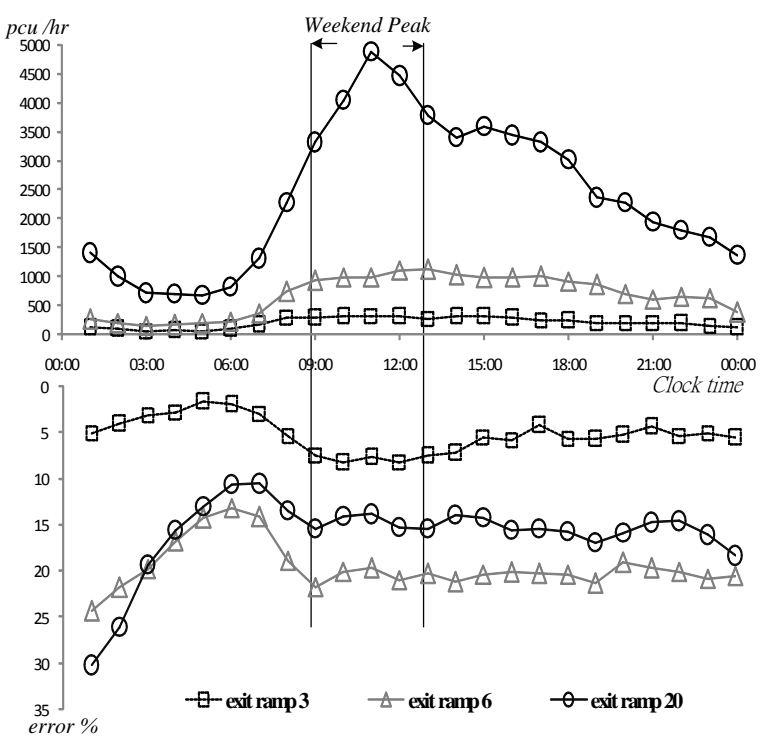

Fig.5

The error distribution and off-ramp flow counts for weekend

\section{Conclusions}

The series studies had established O-D estimation model with explicit time-dimension parameters as time-dependent TDFAM model using in-flow and out-flow data of freeway ramps to estimate O-D flows without executing traditional full-scale equilibrium assignment. However, the structure of simultaneous matrices calculation provides O-D matrices 
estimation in the form of continuity throughout the time span.

Though the structure complicated, the application of TDFAM model on freeway section with large size data, multi-layer matrices, acceptable errors and calculation efficiency showed the practicability of this methodology.

\section{References}

[1] L. G., Willumsen, "Simplified transport models based on traffic counts," Transportation, No. 10, 1981, pp.257-278.

[2] P.-W. Lin, and G.-L. Chang, "A generalized model and solution algorithm for estimation of the dynamic freeway origin-destination matrix," Transportation Research B, Vol. 41, 2007, pp.554-572.

[3] P. Jiao, H. Lu and L. Yang, "Study on Estimation of Dynamic Origin-Destination Flows in Freeway Corridors," International Conference on Networking, Sensing and Control, 2005, pp 948-953.

[4] P. Jiao, "Estimation of Dynamic Network Origin-Destination Flows Using Measured Traffic Counts," Proceedings of the
International Conference on E-Product E-Service and E-Entertainment (ICEEE), Henan, China, November 7-9 2010.

[5] P.-W. Lin and G.-L. Chang, "Robust Estimation of the Dynamic Origin-Destination Matrix for a Freeway Network," International Conference on Networking, Sensing \& Control, Taipei, Taiwan, March 21-23, 2004, pp 862-867.

[6] S. Tsygalnitzky, "Simplified methods in transportation analysis," Thesis Presented to Massachusetts Institute of Technology, at Cambridge, Mass, 1977.

[7] Y.-C. Kao, Estimation of Origin-Destination Matrixes on the Access-Controlled Corridors, Master Thesis, Department of Civil Engineering, Institute of Chung Yuan Christian University , Taiwan, 2005. (In Chinese)

[8] Y.-C. Liao and C.-H. Lin, "The modification of Fluid Analogy Method for O-D Estimation on highway corridors," the $2^{\text {nd }}$ International Conference on Electric Technology and Civil Engineering, Hubei, China, May 18-20, 2012. 